\title{
Multifrequency Polarimetry of CSS Sources
}

\author{
Everton Lüdke, Chidi E. Akujor and Simon T. Garrington \\ NRAI-Jodrell Bank, Cheshire, SK11 9DL, UK
}

Compact Steep Spectrum Sources (CSS) have projected linear sizes $\leq 20 \mathrm{kpc}$ and overall steep spectrum $\left(S \sim \nu^{-\alpha}, \alpha \geq 0.6\right)$. Interactions with a dense interstellar medium may confine the CSSs and produce the high amounts of Faraday rotation that some distorted sources present [1]. The first results of a detailed study of the multifrequency polarization properties of a sample of CSS sources with MERLIN and the VLA are presented.

Some of the CSSs show symmetric brightness distribution with asymmetry in the polarized brightness. Radiogalaxies tend to be less distorted than quasars, with weaker Faraday effects. Quasars which show no integrated polarized flux in low-resolution observations also show no polarized flux in the components, although they often exhibit features like jets, lobes and hotspots, which are associated with the polarized components of extended quasars (e.g. 3C49). The majority of the sources have magnetic field lines colinear with the jet axis (e.g. 3C138), which is similar to the magnetic field configuration of jets in extended radio sources. Comparison with published data at 3.6 and $2 \mathrm{~cm}$ [2] also suggests that the CSSs have larger Faraday dispersions than radio sources with larger linear sizes. This may be the effect of the external hot media denser than in extended sources, thermal gas mixed with source plasma or both, with $\sim 300 \mathrm{pc}$ maximum linear scale for thermal irregularities which depolarize the radio radiation.
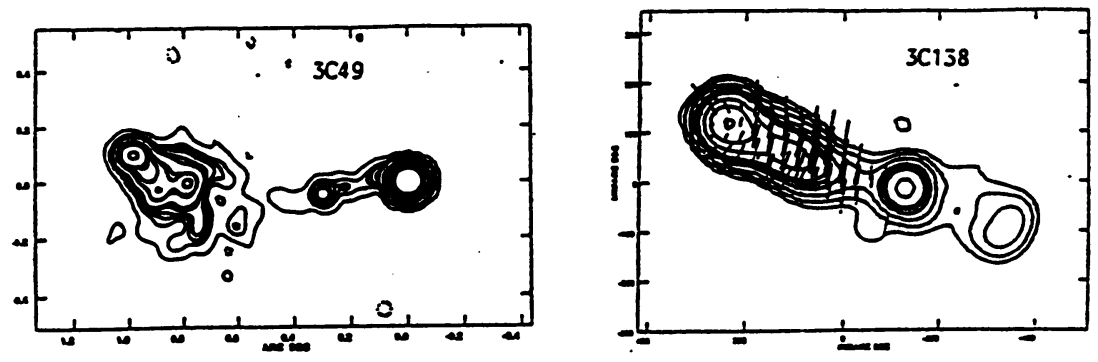

[1] Kato T., Tabara H., Ionue M. and Aizu K., 1987, Nature, 329, 223

[2] van Breugel W.J.M. et al, 1992, A\&A, 256, 56

471

T. J.-L. Courvoisier and A. Blecha: Multi-Wavelength Continuum Emission of AGN, 471.

C 1994 IAU. Printed in the Netherlands. 